\title{
List of contributing authors
}

\section{Tristram Chivers}

Department of Chemistry

University of Calgrary

2500 University Drive NW

Calgary, Alberta

Canada T2N 1N4

Chapter 1, 2, 4

\section{Risto S. Laitinen}

Laboratory of Inorganic Chemistry, Environmental and Chemical Engineering University of Oulu

P.O. Box 3000

90014 Oulu, Finland

Chapter 1, 2, 4

Vimal K. Jain

UM-DAE Centre for Excellence in Basic

Sciences

Health Centre Building

University of Mumbai

Kalina Campus

Santacruz (E), Mumbai-400098 (India)

E-mail: jainvk@cbs.ac.in

Chapter 11

\section{G. Kedarnath}

Chemistry Division

Bhabha Atomic Research Centre

Tromaby, Mumbai-400 085 (India)

and

Homi Bhabha National Insitute

Anushaktinagar, Mumbai-400 094 (India)

E-mail:kedar@barc.gov.in

Chapter 11

\section{Raija Oilunkaniemi}

Laboratory of Inorganic Chemistry,

Environmental and Chemical Engineering

University of Oulu

P.O. Box 3000

90014 Oulu, Finland

Chapter 1

Jamie S. Ritch

Department of Chemistry

The University of Winnipeg

515 Portage Avenue

Winnipeg, Manitoba, R3B 2E9, Canada

j.ritch@uwinnipeg.ca

Chapter 6

\section{Claudio Santi}

Department of Pharmaceutical Sciences

University of Perugia

Perugia, Italy

Chapter 9

\section{Lucia Capoccia}

Department of Pharmaceutical Sciences

University of Perugia

Perugia, Italy

Chapter 9

\section{Bonifacio Monti}

Department of Pharmaceutical Sciences

University of Perugia

Perugia, Italy

Chapter 9 


\section{Alexandra Pop}

Department of Chemistry

Supramolecular Organic and Organometallic Chemistry Centre (SOOMCC)

Faculty of Chemistry and Chemical Engineering Babeş-Bolyai University

11 Arany Janos

400028 Cluj-Napoca, ROMANIA

Chapter 3

\section{Cristian Silvestru}

Department of Chemistry

Supramolecular Organic and Organometallic Chemistry Centre (SOOMCC)

Faculty of Chemistry and Chemical Engineering Babeş-Bolyai University

11 Arany Janos

400028 Cluj-Napoca, ROMANIA

Chapter 3

\section{Anca Silvestru}

Department of Chemistry

Supramolecular Organic and Organometallic Chemistry Centre (SOOMCC)

Faculty of Chemistry and Chemical

Engineering

Babeş-Bolyai University

11 Arany Janos

400028 Cluj-Napoca, ROMANIA

ancas@chem.ubbcluj.ro

Chapter 3

Peter C. Ho

McMaster University

Department of Chemistry and Chemical

Biology

1280 Main Street West

Hamilton, Ontario

Canada, L8S 4M1.

Chapter 8

\section{Jin Wang}

McMaster University

Department of Chemistry and Chemical

Biology

1280 Main Street West

Hamilton, Ontario

Canada, L8S 4M1.

Chapter 8

Ignacio Vargas-Baca

McMaster University

Department of Chemistry and Chemical

Biology

1280 Main Street West

Hamilton, Ontario

Canada, L8S 4M1.

vargas@chemistry.mcmaster.ca

Chapter 8

Fateh V. Singh

Cardiff University

School of Chemistry

Main Building

Park Place, Cardiff

CF10 3AT United Kingdom and

VIT University

Chennai Campus

Vandalur-Kelambakkam Road

Chennai-600127, Tamil

Nadu, India

Chapter 7

Thomas Wirth

Cardiff University

School of Chemistry

Main Building

Park Place, Cardiff

CF10 3AT United Kingdom

wirth@cf.ac.uk

Chapter 7 


\section{Guoxiong Hua}

EaStCHEM School of Chemistry

University of St Andrews

Fife, KY16 9ST, UK

Chapter 5

\section{J. Derek Woollins}

EaStCHEM School of Chemistry

University of St Andrews

Fife, KY16 9ST, UK

Chapter 5
Alexander M. Polgar

Department of Chemistry

Western University

1151 Richmont Street

London, Ontario

Canada N6A 5B7

Chapter 10

John F. Corrigan

Department of Chemistry

Western University

1151 Richmont Street

London, Ontario

Canada N6A 5B7

corrigan@uwo.ca

Chapter 10 
